\title{
An Update: Standardized Hypertension Management to Reduce Cardiovascular Disease Morbidity and Mortality Worldwide
}

\author{
Pragna Patel ${ }^{1 *}$, Pedro Ordunez ${ }^{2}$, Kenneth Connell ${ }^{3}$, Dan Lackland ${ }^{4}$ and Donald DiPette ${ }^{5}$ \\ ${ }^{1}$ Centers for Disease Control and Prevention, Atlanta, GA, USA \\ ${ }^{2}$ Pan American Health Organization, Washington DC, USA \\ ${ }^{3}$ University of the West Indies, Cave Hill Campus, St. Michael, Barbados \\ ${ }^{4}$ Medical University of South Carolina, Charleston, SC, USA \\ ${ }^{5}$ University of South Carolina and University of South Carolina School of Medicine, Columbia, SC, USA
}

*Correspondence author: Pragna Patel, Centers for Disease Control and Prevention, 1600 Clifton Road, Atlanta, GA, 30333, Tel: 404-639-6132, Email: ppatel1@cdc.gov

Received date: April 26, 2017; Accepted date: May 22, 2017; Published date: May 29, 2017

Copyright: (C 2017 Patel P, et al. This is an open-access article distributed under the terms of the Creative Commons Attribution License, which permits unrestricted use, distribution, and reproduction in any medium, provided the original author and source are credited.

Citation: Patel P, Ordunez P, Connell K, et al. An Update: Standardized Hypertension Management to Reduce Cardiovascular Disease Morbidity and Mortality Worldwide. 2017. 3:1.

\section{Short Communication}

Cardiovascular diseases (CVD) are the leading cause of death globally. In 2013, CVD were responsible for approximately $32 \%$ or 17 million deaths worldwide, with $>75 \%$ occurring in low- and middle-income countries (LIMCs) [1]. Hypertension is the leading remediable risk factor for CVD; it affects $>1$ billion people worldwide and is responsible for $>10$ million largely preventable deaths globally each year [1]. Among those with hypertension, $33 \%$ are not aware of their condition and among those who are aware, only $56 \%$ are on treatment [2]. Furthermore, only $28 \%$ of people with hypertension worldwide, have their blood pressure controlled (i.e., at a clinical target of $<140 / 90 \mathrm{mmHg}$ ) [2].

Prevention of CVD with population strategies such as salt reduction, smoking cessation and smoke-free policies, and laws to reduce air pollution are all paramount [3]. Alongside prevention efforts, there will always be a need to specifically address the treatment of hypertension. Improved diagnosis and treatment of hypertension is the most cost-effective means to improve premature mortality in LMICs [4]. However, a major obstacle to hypertension control is the absence of comprehensive primary healthcare services, including limited access to safe and effective medications, and lack of systems to effectively deliver prevention, screening, and treatment. However, building on lessons learned from the treatment of HIV/ AIDS and tuberculosis in LMICs, as well as successful models of hypertension control in the United States and Canada [5-10], the control of hypertension is attainable using a health system strengthening approach. Furthermore, using hypertension as an entry point to strengthen health services, establishes an improved healthcare infrastructure for comprehensive, sustainable management of all chronic conditions.

To meet the challenge of improving the control of hypertension, we encourage Standardized Hypertension
Management (Figure 1) which involves utilizing evidence-based clinical interventions to improve health care delivery. These interventions include the use of standardized treatment protocols, team-based care (task shifting and collaboration), patient empowerment, a core set of medications (formulary) along with improved procurement mechanisms to increase the availability and affordability of these medications, registries for cohort monitoring and evaluation with specific metrics for quality improvement, performance assessment, and community engagement. With political will and strong partnerships, this approach provides the groundwork to reduce the burden of CVD. Additionally, this approach will strengthen primary care delivery and can be applied to other chronic conditions for improved management of noncommunicable diseases (NCDs) overall.

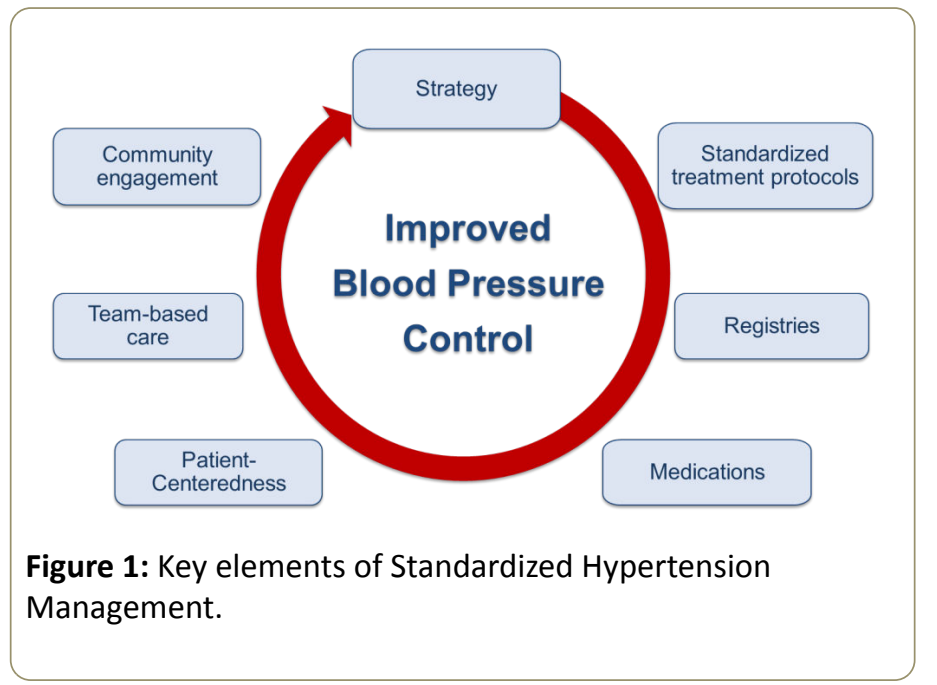

Implementation of this Standardized Hypertension Management approach in the PAHO region, known as the Standardized Hypertension Treatment and Prevention Project (SHTP) [11], has been concluded in Barbados as has been 
recently initiated in Chile, Colombia, and Cuba, as a quality improvement initiative. The SHTP Project, which has all the components recommended by the chronic care model [12], is not promoting the control of hypertension through a vertical program but rather advocates using hypertension as an entry point for comprehensive CVD management. PAHO has made great strides to broaden access to care and availability of medications for NCDs using PAHO's Strategic Fund (www.paho.org\strategicfund) which makes treatment very feasible in this region if countries choose to use the mechanism for medication procurement. However, while the SHTP Project is putting significant emphasis on the management and control of hypertension, an area that has been neglected in many countries, its framework and principles fully support the prevention of CVD/NCDs, particularly through reducing population salt consumption, promoting physical activity and a healthy diet and reducing harmful use of alcohol and exposure to tobacco smoke as well as advocating for smoking cessation. These lifestyle changes are important to address many NCDs and the main co-morbidities commonly seen in patients with hypertension, such as diabetes.

The Caribbean is leading the way with the first SHTP Project pilot in Barbados showing promising results; 18 months post implementation, $>30,000$ persons were screened and the hypertension prevalence in the two pilot sites was 33\%. Notably, there was an increase in blood pressure control rates from $51 \%$ to $66 \%$ over the 18 months of the program. This $15 \%$ increase in blood pressure control is largely attributed to improved prescribing practices, as outlined in the standardized treatment protocols that were adopted. Prescriptions of the suggested first-line medication, chlorthalidone, increased by 3.5\% which also translated to cost-savings for the overall program. As a result, the Barbados Drug Formulary will continue to improve availability of the core set of anti-hypertensive pharmacologic medications, particularly fixed-dose combination pills. Improvements in both systolic and diastolic blood pressure rates were seen across all age groups but were particularly prominent among persons $>60$ years of age. However, among women, the most significant declines were seen in 50-69 year olds and among men, the most significant declines were seen among 20-49 and $>70$ year olds. The program plans to explore how to improve management of hypertension among middle-aged men, a challenge that is also prominent in the United States. More than one-third of Barbadians aged 25-70 years suffer from hypertension, therefore the lessons learned and positive outcomes of this project will be disseminated and plans for national expansion in Barbados are underway (https:// www.youtube.com/watch?v=bQ7j4g5qZVs).

The Healthy Caribbean Coalition (HCC) was instrumental to the success of the Barbados pilot and further extended the principles of Standardized Hypertension Management to five Caribbean countries in collaboration with the University of the West Indies. The SHTP has been a key feature in the HCC's Direct Aid Program Part 2's Blood Pressure Control through Community Action Project (BPCCA). One-day community based 'Hypertension Screening \& Monitoring' workshops were conducted in Dominica, Haiti, Jamaica, St. Lucia, and St. Vincent. SHTP principles and experiences were shared at these workshops and were very well received, particularly; the SHTP's standardized hypertension treatment protocol was appreciated and recognized as a useful tool. Several other countries in the PAHO region have expressed interest in SHTP including Antigua, the Bahamas, Curacao, Ecuador, Jamaica, and St. Lucia.

Regional expansion has also been undertaken. Chile, Colombia, and Cuba are implementing a comprehensive and innovative approach aligned with the PAHO NCD agenda to reduce CVD risk through hypertension control and secondary prevention. This approach, inspired by the SHTP Project and as well as lessons learned from the pilot in Barbados, uses four main interventions: introducing simple, standardized, evidencebased treatment algorithms, improving the availability and access to a core set of high quality medications, and instituting a clinical registry for patient monitoring and for evaluating healthcare organization performance. This approach complements and reinforces current efforts in CVD/NCD prevention in these countries, but concurrently emphasizes the comprehensive management of hypertension to improve care. Health systems strengthening is needed as evidenced by the low levels of blood pressure control and poor coverage of secondary prevention, both critical interventions to reduce the premature mortality due CVD, in the region.

The ultimate goal of the SHTP demonstration projects was to strengthen existing health systems such that other chronic diseases can also be addressed. Lessons learned from these countries suggest that to effectively reduce the burden of hypertension within a care delivery system, it is essential to have strong policies, political will, and effective partnerships. In this vain, the World Hypertension League advocates for strategic planning by national and regional organizations using hypertension as a focus and endorses the Standardized Hypertension Management approach [13].

Standardized Hypertension Management complements population approaches to primary prevention. In combination with salt reduction strategies, improved blood pressure control may avert $>10$ million deaths and adverse events due to CVD [14]. Furthermore, the interventions supported by this approach are invaluable for a necessary paradigm shift to adapt health systems that have historically focused care on acute infectious illnesses for the practice of preventive medicine and for the long-term management of chronic diseases. Thus, we believe that Standardized Hypertension Management will enhance health care delivery to reduce CVD burden worldwide.

The Standardized Hypertension Treatment and Prevention Project pilots were undertaken as a proof-of-concept to evaluate the feasibility of implementing evidence-based clinical interventions at the primary health care level. The aim was to strengthen health systems and to improve the quality of care of NCDs, specifically CVD, using hypertension as an entry point, in LMICs. The results of the pilot sites were promising with increases in blood pressure control rates, development of registries for improved care, and improved prescribing practices. Key elements of the Standardized Hypertension Management approach including the use of standardized treatment algorithms, improved access to medications and improved clinical monitoring systems have now been included in a more 
comprehensive global initiative for CVD prevention and treatment. The Global HEARTS initiative to reduce heart attack and strokes will support the implementation of the HEARTS technical package, a set of six technical and operational interventions to scale-up CVD management at the primary healthcare level. These include Healthy lifestyle counselling, Evidence-based treatment protocols, Access to essential medicines and technologies, Risk based management, Team based care and task sharing and Systems for monitoring. The Global HEARTS Initiative is being led by the World Health Organization in collaboration with the U.S. Centers for Disease Control and Prevention, PAHO, the World Heart Federation, the World Stroke Organization, the International Society of Hypertension, the World Hypertension League and other partners [15].

\section{Acknowledgements}

We would like to acknowledge the Standardized Hypertension Treatment and Prevention Network for their contributions to the development of this framework. Members include: Alma Adler, World Heart Federation; Sonia Y. Angell, New York City Department of Health and Mental Hygiene; Samira Asma, CDC; Michele Bashin, Public Health Institute; Rafael Bengoa, Minister of Health, Basque Region; Ana Isabel Barrientos Castro, Sociedad Interamericana de Cardiología and Sociedad Centroamericana de Cardiología; Barbara Bowman, CDC; Francis Burnett, Organization of Eastern Caribbean States; Jose Castro, PAHO; Norman Campbell, World Hypertension League; Beatriz Marcet Champagne, InterAmerican Heart Foundation; Kenneth Connell, University of West Indies; Jose De Gracia, Martin Didier, Caribbean Cardiac Society; Donald DiPette, University of South Carolina; Maria Cristina Escobar, Chile Ministry of Health; Daniel Ferrante, Argentina Ministry of Health; Thomas Gaziano, Harvard School of Public Health; Marino Gonzalez, Universidad Simon Bolivar; Sir Trevor Hassell, Health Caribbean Coalition; Anselm Hennis, PAHO; Rafael Hernandez, Universidad Centroccidental Lisandro Alvarado, Venezuela; Maryam Hinds, Barbados Drug Service; Marc Jaffe, Kaiser Permenante; Fernando Lanas, Universidad de La Frontera, Chile; Patricio Lopez-Jaramillo, Latin American Society of Hypertension; Fleetwood Loustalot, CDC; Ben Lumley, Public Health England; Javier Maldonado, Colombia Ministry of Health; Thelma Nelson, National Health Fund; Jose Miguel do Nascimento Junior, Brazil Ministry of Health; Pedro Ordunez, PAHO; Marcelo Orias, Latin American Society of Nephrology and Hypertension; Jose Ortellado, Latin American Society of Internal Medicine; Pragna Patel, CDC; Jacqueline Poselli, Venezuela Foro Farmaceutico de Las Américas; Agustin Ramirez, International Society of Hypertension; Lynn Silver, Public Health Institute; Donald Simeon, Caribbean Cardiac Society; Valerie Steinmetz, Public Health Institute; Kathryn Taubert, ; Honorable Alvina Reynolds, St. Lucia Ministry of Health; Hilary Wall, CDC; Jamie Waterall, Public Health England; Fernando Wyss, Interamerican Society of Cardiology; Amy Valderrama, CDC; Jose Fernando Valderrama,
Colombia Ministry of Health; and Fernando Lanas Zanetti, Interamerican Society of Cardiology.

\section{References}

1. GBD 2013 Risk Factors Collaborators (2015) Global, regional, and national comparative risk assessment of 79 behavioural, environmental and occupational, and metabolic risks and clusters of risks in 188 countries, 1990-2013: A systematic analysis for the Global Burden of Disease Study 2013. Lancet.

2. Mills KT, Bundy JD, Kelly TN, Reed JE, Kearney PM, et al. (2016) Global disparities of hypertension prevalence and control: a systematic review of population-based studies from 90 countries. Circulation 134: 441-450.

3. First Global Ministerial Conference on Healthy Lifestyles and Non communicable Disease Control (2011) Moscow.

4. Nugent R (2015) Benefits and Costs of the Non communicable Disease Targets for the Post- 2015 Development Agenda. Copenhagen Consensus Center.

5. Jaffe MG, Lee GA, Young JD, Sidney S, Go AS (2013) Improved Blood Pressure Control Associated With a Large-Scale Hypertension Program. J Am Med Assoc 310: 699-705.

6. McAlister FA, Wilkins $K$, Joffres $M$, Leenen FH, Fodor $G$, et al. (2011) Changes in the rates of awareness, treatment and control of hypertension in Canada over the past two decades. CMAJ 183: 1007-1013.

7. Harries AD, Zachariah R, Jahn A, Schouten EJ, Kamoto K (2009) Scaling up antiretroviral therapy in Malawi - implications for managing other chronic diseases in resource-limited countries. J Acquir Immune Defic Syndr 52

8. Seita A, Harries AD (2013) All we need to know in public health we can learn from tuberculosis care: lessons for non-communicable disease. Int J Tuberc Lung Dis 17: 429-430.

9. Frieden TR, Berwick DM (2011) The "Million Hearts" initiative preventing heart attacks and strokes. N Engl J Med 365.

10. Jafar HT, Hatcher J, Poulter N, Islam M, Hashmi S, et al. (2009) Community-based interventions to promote blood pressure control in a developing country. Ann Intern Med 151: 593-601.

11. Patel P, Ordunez P, DiPette D, Escobar MC, Hassell T, et al. (2016) Improved blood pressure control to reduce cardiovascular disease morbidity and mortality: The Standardized Hypertension Treatment and Prevention Project. J Clin Hypertens.

12. Wagner EH, Austin BT, Davis C, Hindmarsh M, Schaefer J, et al. (2001) Improving chronic illness care: Translating evidence into action. Health Aff 20: 64-78.

13. Campbell NR, Lackland DT, Lisheng L, Zhang X, Nilsson PM, et al. (2015) The World Hypertension League Challenges Hypertension and Cardiovascular Organizations to Develop Strategic Plans for the Prevention and Control of Hypertension. J Clin Hypertens 17: 325-327.

14. Angell SY, DeCock KM, Frieden TR (2015) A public health approach to global management of hypertension. Lancet 385: 825-827.

15. World Health Organization. Global Hearts Initiative (2016). 